\title{
STEM-EELS Imaging of Resonant Modes in Dielectric Silicon Nanostructures
}

\author{
Valentin Flauraud ${ }^{1}$ and Duncan T.L. Alexander ${ }^{*}$ \\ 1. Electron Spectrometry and Microscopy Laboratory (LSME), Institute of Physics (IPHYS), Ecole \\ Polytechnique Fédérale de Lausanne (EPFL), Lausanne, Switzerland. \\ * Corresponding author: duncan.alexander@epfl.ch
}

In recent years, the field of plasmonics has stimulated intensive research for diverse applications, with electron energy-loss spectroscopy (EELS) based techniques becoming a primary tool for high spatial resolution near-field mapping of plasmonic eigenmodes [1]. However, strong absorption losses limit the performance of metallic plasmonic nanostructures for various nanophotonic applications. A promising strategy to overcome this is to use electric and magnetic Mie-type resonances in dielectric nanoparticles [2]. Compared to metallic plasmonic excitations, these resonances present some key differences [3]: they are driven by displacement currents rather than actual currents (hence their low optical absorption losses); the charge displacement and hence resonant eigenmodes are supported within the volume of the nanoparticle, rather than on the surface; in addition to the excitation of electric modes, the driving of displacement current loops can produce magnetic resonant modes. Until now, sub-wavelength imaging of these resonant modes has been limited to near field optical microscopy, e.g. [4], and a few studies using cathodoluminescence (CL), e.g. [5, 6]. Here, we report on the first EELS studies of resonant eigenmodes in silicon dielectric nanoparticles of varying size and geometry.

The samples are prepared by electron lithographic patterning of Si nanostructures $\sim 100 \mathrm{~nm}$ high on silicon nitride windows in 4-inch silicon wafers, with each wafer containing 256 "transmission electron microscopy (TEM)-ready" squares which can be cleaved out and inserted in the TEM sample holder. The structures are measured using a FEI Titan Themis 60-300 equipped with a Gatan GIF Quantum ERS spectrometer. To help measure the modes within the projected particle volume, a $300 \mathrm{kV}$ high tension is applied to minimize scattering to the bulk plasmon. Combining a high incident beam current of 200-250 pA, monochromated for an energy spread of 100-110 meV FWHM, and fast scanning TEM (STEM)EELS mapping $\left(>1000\right.$ pixels $\mathrm{s}^{-1}$ ) with the patterned samples allows the systematic analysis of tens of Si structures in function of particle shape and size. Here we describe a selection of the results recorded.

Fig 1(a, b) presents intensity maps of different modes excited in Si discs of 100 and $300 \mathrm{~nm}$ diameters, along with EELS spectra from different impact parameters. Both discs display lowest energy excitations of in-plane dipole modes, that are at a lower energy/longer wavelength in the larger $300 \mathrm{~nm}$ disc, in which higher order planar modes are also excited. The $300 \mathrm{~nm}$ disc also shows a vertical electric dipole mode at $\sim 2.35 \mathrm{eV}$ energy loss, excited most strongly in the nanoparticle center [3], analogous to the "breathing mode" excitation seen in plasmonic nanoparticles. As disc diameter increases further, two inplane dipole modes can be discriminated. For instance, a $450 \mathrm{~nm}$ disc shows dipoles at 1.01 and $1.36 \mathrm{eV}$ energy loss, which we expect correspond to the electric and magnetic dipoles, respectively [7]. Figure 1(c) presents results for a $450 \times 300 \mathrm{~nm}$ ellipse. The lack of radial symmetry breaks the degeneracy of the in-plane modes, leading to separate lateral and vertical dipole modes, and a variety of complex eigenmodes between $\sim 1.5-2.2 \mathrm{eV}$ energy loss. Interestingly, the $1.74 \mathrm{eV}$ mode is similar to one seen for a $450 \times 300 \mathrm{~nm}$ rectangle, and so seems characteristic of aspect ratio, while the strong $1.55 \mathrm{eV}$ mode is like one seen for a $600 \times 300 \mathrm{~nm}$ ellipse, and so seems related to particle shape. These observations could be connected to whether the corresponding excitations are electric or magnetic in nature. 
Theoretical work is currently underway to simulate and interpret the data presented here. Compared to published data on resonant modes in silicon nanoparticles from scanning electron microscopy-based CL, e.g. [5, 6], the STEM-EELS technique covers a larger spectral range, and the different modes are spatially resolved with high definition and low noise. On a theoretical level, while CL can only observe "leaky" modes in which the field propagates outside the structure, the penetrating nature of EELS should be capable of measuring excitations in which the field is entirely confined within the nanoparticle. It therefore presents a promising avenue for exploring resonant modes in dielectric systems [8].

\section{References:}

[1] FJ García de Abajo, Rev. Mod. Phys. 82 (2010), p. 209.

[2] M. Decker and I. Staube, J. Opt. 18 (2016), 103001.

[3] J van de Groep and A Polman, Opt. Express 21 (2013), 26285.

[4] TF Habteyes et al., ACS Photonics 1 (2014), p. 794.

[5] T Coenen, J van de Groep and A Polman, ACS Nano 7 (2013), p. 1689.

[6] J van de Groep et al., Optica 3 (2016), 93.

[7] I Staude et al., ACS Nano 7 (2013), p. 7824.

[8] We thank the CIME and CMI at EPFL for access to their facilities.

(a) $100 \mathrm{~nm}$ dia. disc
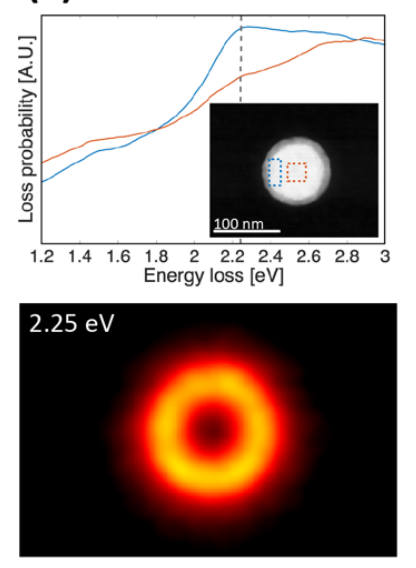

(b) $300 \mathrm{~nm}$ diameter disc
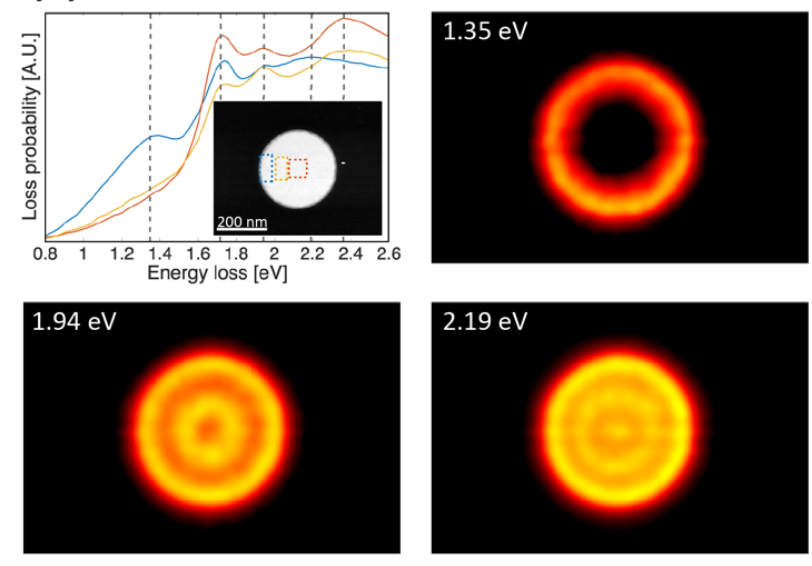

(c) $450 \times 300 \mathrm{~nm}$ ellipse
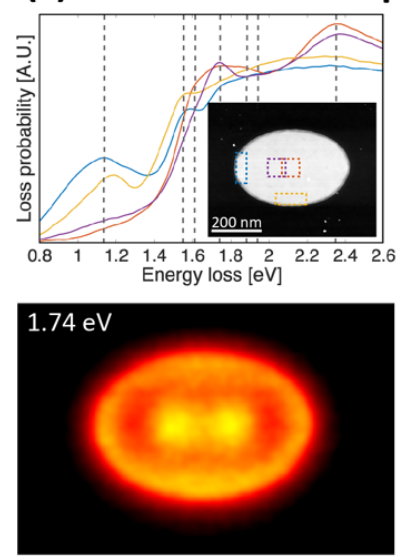
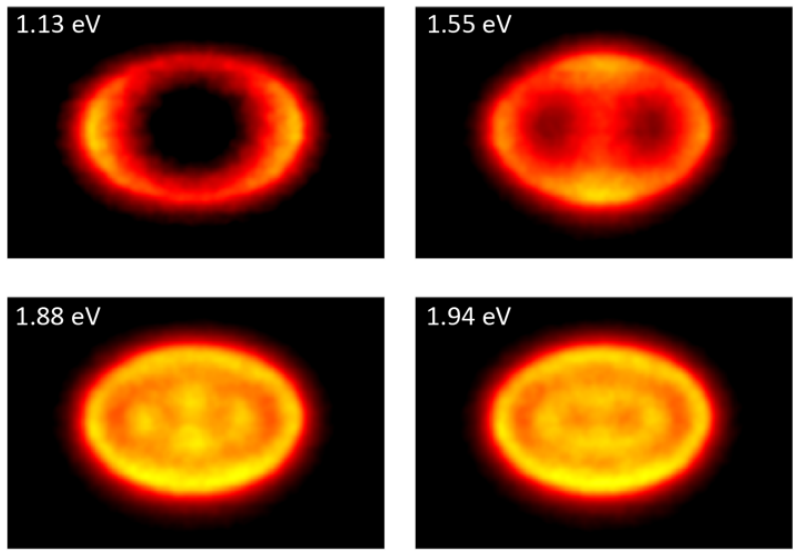

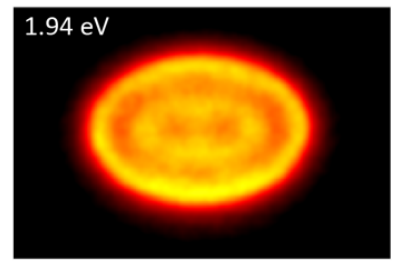

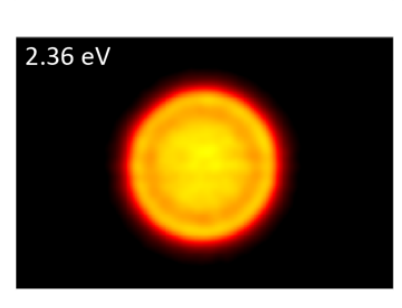
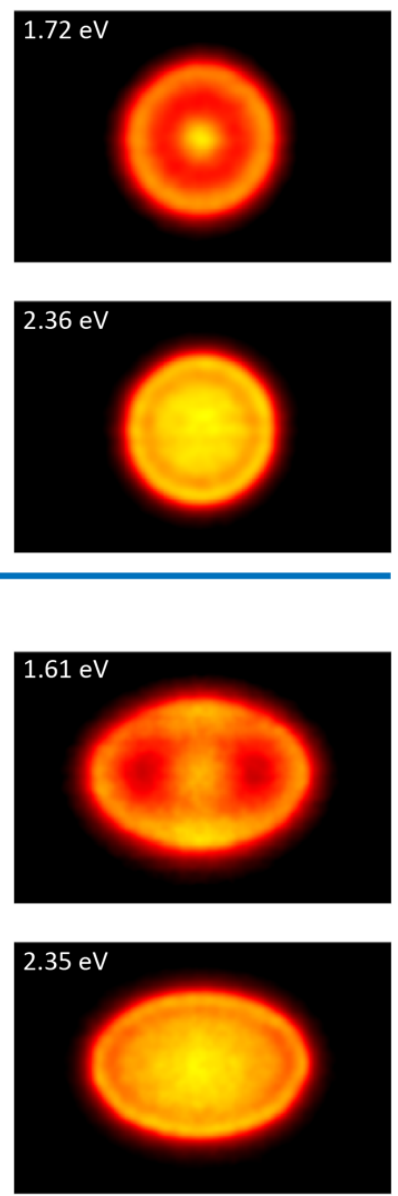

Figure 1. EELS spectra and heat intensity maps of resonant eigenmodes in Si nanoparticles. EELS spectra are integrated from regions indicated on HAADF images of the particles with color-coded boxes. 